\title{
A Novel FET-type Hydrogen Gas Sensor with Pd-decorated Single-Walled Carbon Nanotubes by Electroplating Method
}

\author{
Seongbin Hong, Jongmin Shin, Yoonki Hong, Meile Wu, Yujeong Jeong, Jong-Ho Bae, Jong-Ho Lee* \\ Department of Electrical and Computer Engineering and Inter-university Semiconductor Research \\ Center, Seoul National University, Seoul, 08826, Republic of Korea \\ *jhl@snu.ac.kr
}

\begin{abstract}
In this work, we investigate the hydrogen $\left(\mathrm{H}_{2}\right)$ gas sensing performance in a $p$-type FET sensor having a floating-gate $(F G)$ and a control-gate (CG) placing each other. Single-walled carbon nanotubes (SWNTs) are formed between the CG and FG by an inkjet-printing method to be used as a sensing layer. Then, SWNTs are decorated with palladium (Pd) by an electroplating method. The $\mathrm{H}_{2}$ gas responses are studied by measuring the transfer characteristics (ID- $V_{C G}$ ) and transient currents (ID) with different $\mathrm{H}_{2}$ concentrations under various $C G$ biases $\left(V_{C G S}\right)$. As the $\mathrm{H}_{2}$ concentration increases from $0.02 \%$ to $1 \%$, the sensitivity of $\mathrm{H}_{2}$ gas increases and then starts to saturate regardless of operation region of FET-type sensor. The saturation is caused by a decrease in work-function, resulting from the diffusion of $\mathrm{H}_{2}$ and the generation of $\mathrm{H}_{2} \mathrm{O}$.
\end{abstract}

Keywords: $\mathrm{H}_{2}$ gas sensor, FET, SWNTs, Pd, electroplating

\section{Device fabrication}

The structure of the fabricated sensor is shown in the cross-sectional schematic views and the optical microscopic image of the device shown in Fig. 1(a) and (b), respectively. Here, the key fabrication processes of the sensor are explained. A $n$-type Si (100) wafer is used as a substrate and the active area is formed by LOCOS process. After the gate oxide is thermally grown on the channel, formation of an $n^{+}$ doped polysilicon $F G$ is followed by $O / N / O$ stack. Then a $\mathrm{Cr} / \mathrm{Au} \mathrm{CG}$ is formed. The FG and CG face each other in the form of interdigitated pattern. SWNTs as a sensing layer are formed in the area where the $O / N / O$ stack covering the $F G$ and the $C G$ are interdigitated by an inkjet-printing method. Then, SWNTs are decorated with $\mathrm{Pd}$ by an electroplating method, which is carried out by using $\mathrm{PdCl}_{2}$ as a precursor under $-1 \mathrm{~V}$ of potential for $20 \mathrm{sec}$.

\section{Material characterization}

Fig. 2 shows the energy dispersive X-ray spectroscopy (EDS) spectrum of the sensing layer (Pd-SWNTs). It shows X-ray peaks that indicate the constituent elements of the sensing layer. Pd La and $\mathrm{L} \beta$ peaks are observed at $2.838 \mathrm{keV}$ and $2.990 \mathrm{keV}$, which are similar to those of theoretical values. Table in Fig. 2 show the atomic and weight concentrations of each element.

\section{Results}

Gas sensing characteristics of the fabricated sensor is measured at room temperature $\left(25^{\circ} \mathrm{C}\right)$ in an enclosed chamber. Fig. 3(a),(b) show the transfer characteristics ( $\left.I_{D}-V_{C G}\right)$ of the fabricated FET-type sensor obtained by DC and pulsed measurements, respectively. In Fig. 3(b), the ambience is air and $\mathrm{H}_{2}$ gas, and pulse scheme for pulse measurement is shown in the inset. As the $\mathrm{H}_{2}$ concentration increases from $0.05 \%$ to $1 \%$, the transfer curve is shifted to the left. When the sensor is exposed to $\mathrm{H}_{2}$ gas, $\mathrm{H}_{2}$ molecules are dissociated on the $\mathrm{Pd}$ surface. Then, $\mathrm{H}$ atoms are adsorbed on the Pd surface and quickly diffuse to the $\mathrm{Pd} / \mathrm{CNT}$ interface, resulting in decreasing the work-function of CNTs. Also, $\mathrm{O}_{2}$ in air ambience is reacted with $\mathrm{H}$ atom to form a hydroxyl $(\mathrm{OH})$ group. $\mathrm{OH}$ group finally causes the generation of $\mathrm{H}_{2} \mathrm{O}$ with extra $\mathrm{H}$ atom [1]. Then, the work-function of CNTs decreases, because $\mathrm{H}_{2} \mathrm{O}$ is known as electron donor. In the higher $\mathrm{H}_{2}$ concentration, however, excessive $\mathrm{H}_{2} \mathrm{O}$ causes the Grotthuss chain reaction [2], and it suppresses increase of sensitivity. Fig. 4 (a)-(f) show the transient responses (ID) with different $\mathrm{H}_{2}$ concentrations in the subthreshold and linear regions of the sensor. As the $\mathrm{H}_{2}$ concentration increases from $0.02 \%$ to $1 \%$, sensitivity $\left(\Delta /_{D} / I_{D} \times 100\right)$ of $\mathrm{H}_{2}$ gas increases and starts to saturate around $\mathrm{H}_{2}$ concentration of $0.1 \%$ regardless of operation region of FET-type sensor. It is shown that these results coincide with Langmuir isotherm model [3].

\section{References}

[1] T.-C. Lin, B.-R. Huang, Palladium nanoparticles modified carbon nanotube/nickel composite rods $(\mathrm{Pd} / \mathrm{CNT} / \mathrm{Ni})$ for hydrogen sensing, Sensors and Actuator B: Chemical 162, 108-113 (2012); doi:10.1016/j.snb.2011.12.044

[2] H. Farahani, R. Wagiran, M.N. Hamidon, Humidity Sensors Principle, Mechanism, and Fabrication Technologies: A Comprehensive Review, Sensors 14, 7881-7839 (2014); doi:10.3390/s140507881

[3] Y. Morita, K.-I. Nakamura, C. Kim, Langmuir analysis on hydrogen gas response of palladium-gate FET, Sensors and Actuator B: Chemical 33, 96-99 (1996); http://doi.org/10.1016/0925-4005(96)01956-9 


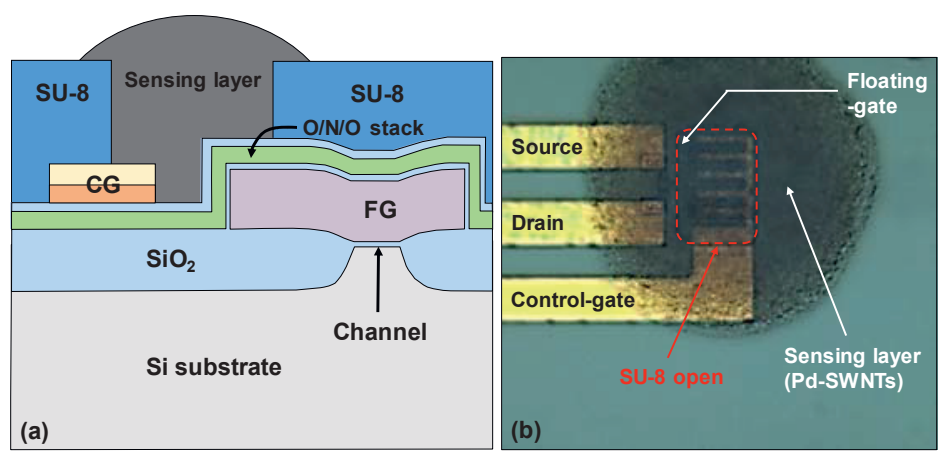

Fig. 1. (a) Cross-sectional schematic view cut along the channel width direction and (b) top optical image of the fabricated sensor.

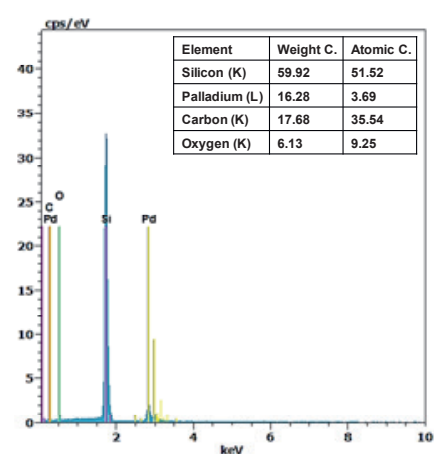

Fig. 2. EDS spectrum of the sensing layer (Pd-SWNTs).
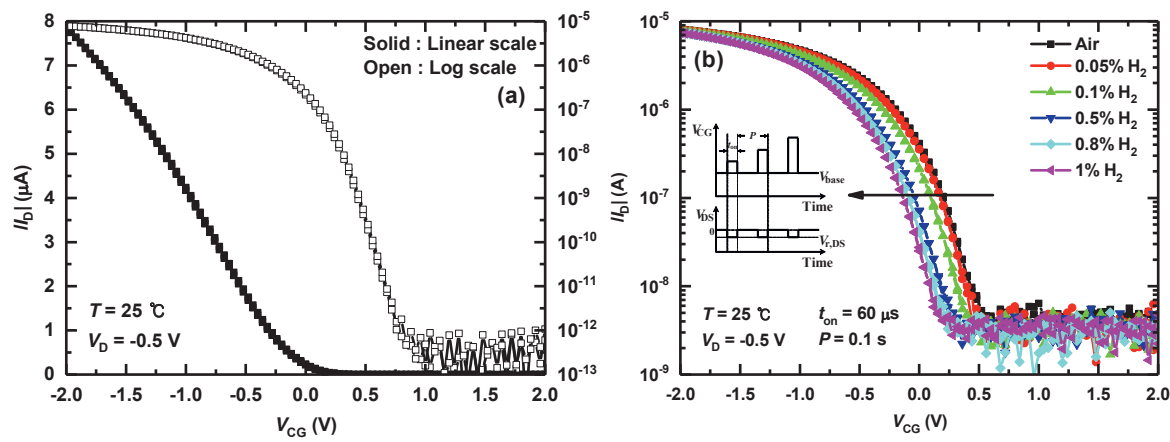

Fig. 3. (a) Transfer characteristics $\left(I_{D}-V_{C G}\right)$ of the fabricated FET-type sensor obtained by DC measurement. (b) $I_{D}-V_{C G}$ curves as a parameter of $\mathrm{H}_{2}$ gas concentration obtained by pulsed measurement (Reference : air).

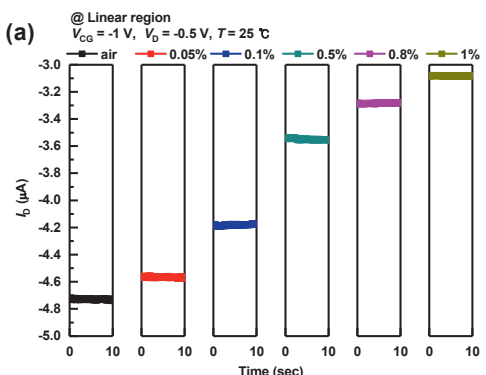

(d) @ Linear region $V_{\mathrm{CG}}=-0.9 \mathrm{~V}, V_{\mathrm{D}}=-0.5 \mathrm{~V}, T=25 \mathrm{r}$

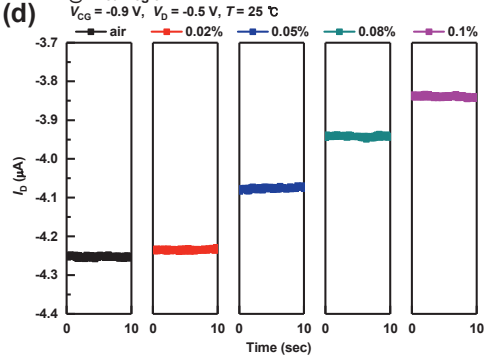

(b) $\begin{aligned} & @ \text { Subthreshold region } \\ & V_{\mathrm{cu}}=0.2 \mathrm{v}, V_{\mathrm{o}}=0.5 \mathrm{~V}, T=25 \mathrm{C}\end{aligned}$

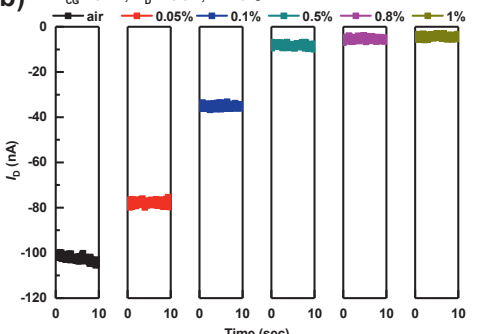

(e) $\mathrm{V}_{\mathrm{cG}}=-0.1 \mathrm{~V}, V_{\mathrm{D}}=-0.5 \mathrm{~V}, T=25 \mathrm{c}$

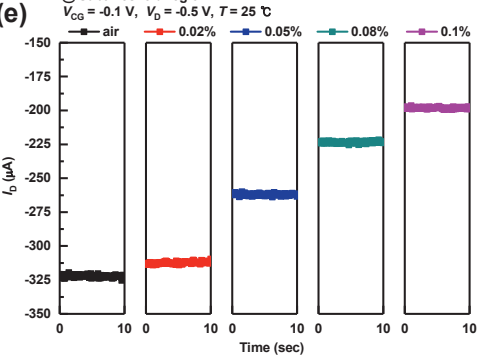

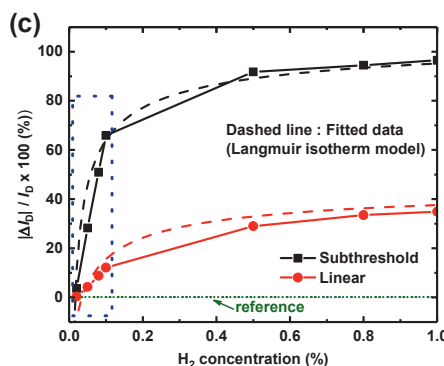

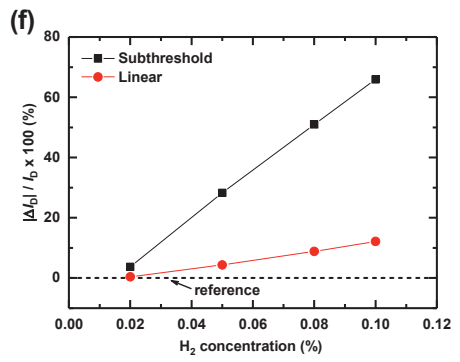

Fig. 4. (a) Transient (ID) behaviors of the fabricated FET-type sensor as a parameter of $\mathrm{H}_{2}$ concentration from $0.05 \%$ to $1 \%$ in linear region and (b) subthreshold region. (c) $\mathrm{H}_{2}$ gas sensitivities versus $\mathrm{H}_{2}$ concentration from $0.02 \%$ to 1\%. Dashed lines are fitted lines by Langmuir isotherm model. (d) Transient (ID) behaviors of the fabricated FET-type sensor as a parameter of $\mathrm{H}_{2}$ concentration from $0.02 \%$ to $0.1 \%$ in linear and $(e)$ subthreshold regions. (f) Magnified plot of blue dotted rectangle $\left(0.02 \% \sim 0.1 \% \mathrm{H}_{2}\right)$ in $(c)$. Measurements are carried out by applying pulses to the control-gate. (Reference : air)

\section{Acknowledgements}

This work was supported in part by the Brain Korea 21 Plus Project in 2018, the National Research Foundation of Korea under Grant NRF-2016R1A2B3009361. 